\title{
Noise induced synchronization of time-delayed semiconductor lasers and authentication based asymmetric encryption.
}

\begin{abstract}
In this work, we propose to enable security mechanisms on a chaotic communication system based upon common noise induced synchronization between two time-delayed semiconductor laser systems. The cryptosystem subjected to the common additive Gaussian colored noise undergoes a transition to follow identical trajectories. An investigation of the system together with a novel scheme for authentication based message encryption process are presented. The encrypted message is also sent over a public channel, while the key is never transmitted at all. The advantage of the scheme is its security, based on the authentication and asymmetric encryption. Extended statistical tests with the proposed two phase cryptography scheme demonstrate the efficiency of the system being robust and tolerant to different types of statistical attacks.
\end{abstract}

Keyword: Noise induced chaos synchronization; Semiconductor laser system; Asymmetric cryptography. 